East African Medical Journal Vol. 83 No. 1 January 2006

THE ROLE OF NEURO-ELECTROPHYSIOLOGICAL DIAGNOSTIC TESTS IN CLINICAL MEDICINE

S.O. Mbuya, MBChB, MMed, (Intern. Med) (Nbi) CTM, Neurologist, Lecturer, Department of Clinical Pharmacology, College of Health Sciences, University of Nairobi, P.O. Box 19994-00202, Nairobi, Kenya

\title{
THE ROLE OF NEURO-ELECTROPHYSIOLOGICAL DIAGNOSTIC TESTS IN CLINICAL MEDICINE
}

\section{S.O. MBUYA}

\begin{abstract}
Objective: To summarise and discuss the role of neuro-electrophysiological diagnostic tests in clinical medicine.

Data Sources: Published original research and reviews to date.

Study Selection: The review was with emphasis on diagnosis of peripheral neuropathic and neuromuscular disorders.

Data extraction and synthesis: On-line and manual library searches provided information and data on which the summaries and discussions were based. Some physicians are not yet aware of some of the tests and some may not know their indications. In this article a review is made of evoked potential tests, nerve conduction tests and electromyography in diagnosis of neurological diseases. An attempt has been made to clearly show their indications, and relative importance.

Conclusion: Studies clearly show that neuro-electrophysiological tests are important in neurological diagnosis in clinical medicine and are an extension of the physical examination.
\end{abstract}

\section{INTRODUCTION}

Neurological diagnosis like in any other medical field involves careful and full history taking, thorough physical examination and investigation. Because of the complex nature and anatomy of the nervous system many neurological diseases are manifested at sites far from the site of injury, for example, lacunar lesion in internal capsule causing monoplegia of one arm. There is need to work out the anatomical site of the lesion. Weakness in a limb could be due to a muscular disease, a nerve disease or both. There is need to distinguish muscular from nerve disease. Medical diseases, metabolic disorders and drug toxicities may cause preferential damage to either axons or myelin sheath, motor nerve or sensory nerves. There is need therefore to know whether it is a motor or sensory disorder. Some upper motor neuron diseases may present with signs and symptoms like lower motor neurons. There is need therefore to know whether disease involves upper motor or lower motor neurons or both, and to know whether the whole limb or only a group of muscles is involved. The clinical diagnosis must be clear. The diagnoses of neurological diseases are at three levels:

(i) Clinical diagnosis, for example, monoplegia of the right arm.

(ii) Anatomical diagnosis, for example, lacunar lesion in the internal capsule.

(iii) Pathological diagnosis, for example, lacunar lesion due to arteritis of polyarteritis nodosa.

Electrophysiological tests are necessary to make an accurate diagnosis. The following are the currently commonly used electrophysiological tests:
1. Evoked Potentials

(a) Visual Evoked Potentials (VEP)

(b) Cognitive Evoked Potentials

(c) Motor Evoked Potentials

(d) Somatosensory Evoked potentials (SSEP)

(e) Auditory Evoked potentials (BAEP)

(f) Blink reflexes

2. Nerve Conduction Studies - Motor/Sensory

3. Electromyography (EMG)

4. Electroencephalography (EEG)

Electromyography is first discussed with its indications and findings in various diseases and the technique briefly explained. This is followed by a discussion of the various nerve conduction studies giving criteria for diagnosing axonal loss and demyelination. The indications are given and techniques explained followed by a brief discussion of how the tests can help in clinching the pathological diagnosis. Lastly the evoked potential tests listed above are briefly discussed explaining the techniques and findings in various diseases.

Electromyography: In electromyography, electrical changes in muscle evoked by voluntary movement are recorded by an electromyography machine. The recording is made by introducing a concentric needle electrode into the muscle to be tested.

When a normal muscle is completely relaxed no electrical activity can be detected in it. When voluntary contraction is induced the record shows the characteristic response of a motor unit which is 
usually biphasic, that, is, shows a positive followed by a negative wave phase. As the contraction increases in strength more motor units contract and more motor unit action potentials (MUAPs) are added to the record $(1,2)$ (Figure 1).

Lessions of the lower motor neuron or muscle produce typical effects.
Denervation: Complete denervation clinically produces flaccid paralysis, muscle wasting with time and loss of reflexes, while partial denervation produces various degrees of weakness. On the EMG denervation leads to appearance of fibrillation potentials, positive sharp waves and fasciculation potentials at rest (Figures 2-4).

Figure 1

Normal motor unit potentials
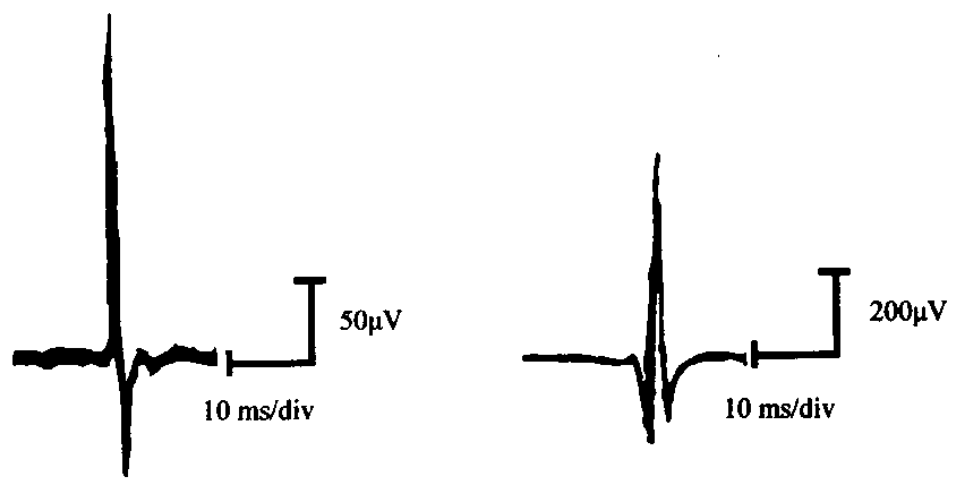

\begin{tabular}{|ccccc|}
\hline AMPLITUDE & DURATION & WAVE FORM & FREQUENCY & SOUND \\
\hline $300-5,000 \mu \mathrm{V}$ & $3-12 \mathrm{~ms}$ & $2-3$ phases & $1-15 \mathrm{~Hz}$ & $\begin{array}{c}\text { Clear } \\
\text { Distinct } \\
\text { "thump" }\end{array}$ \\
\hline
\end{tabular}

Figure 2

Fibrillation potentials


\begin{tabular}{|ccccc}
\hline AMPLITUDE & DURATION & WAVE FORM & FREQUENCY & SOUND \\
\hline $10-600 \mu \mathrm{V}$ & $1-2 \mathrm{~ms}$ & 2 phases & $2-30 \mathrm{~Hz}$ & $\begin{array}{c}\text { High } \\
\text { Pitched } \\
\text { "click" }\end{array}$ \\
\hline
\end{tabular}


Figure 3

Positive sharp wave potentials

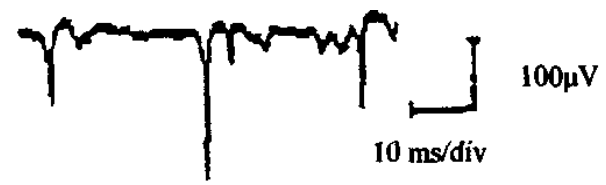

\begin{tabular}{|ccccc|}
\hline AMPLITUDE & DURATION & WAVE FORM & FREQUENCY & SOUND \\
\hline $60-4,000 \mu \mathrm{V}$ & $2-10 \mathrm{~ms}$ & 2 phases & $2-100 \mathrm{~Hz}$ & $\begin{array}{c}\text { Dull } \\
\text { "Thud" }\end{array}$ \\
\hline
\end{tabular}

Figure 4

Fasciculation potentials



\begin{tabular}{|cllll}
\hline AMPLITUDE & DURATION & WAVE FORM & FREQUENCY & SOUND \\
\hline Variable & Variable & Variable & Variable & "Pop" \\
\hline
\end{tabular}

Fibrillation potentials are sharp waves of short duration. Fasciculation potentials are normal in appearance and occur only occasionally. On activity, units are reduced in number and are generally normal in appearance, though in severe chronic denervation many surviving units are increased in amplitude and duration. The characteristic finding-in alpha motor neuron disease is spontaneous fibrillation on mechanical stimulation by the exploring needle, a duration and amplitude of action potentials greater than normal and even in cases of moderate weakness a marked reduction in the number of motor neurons and consequently of the motor units available for contraction.

Myopathy: The characteristic response to voluntary movement is the occurrence of spike potentials with abnormally short duration and much weaker than the normal action potential and an increased proportion of polyphasic action potentials (Figure 5). The number of action potentials is reduced only when there is severe weakness.

Figure 5

Polyphasic potentials

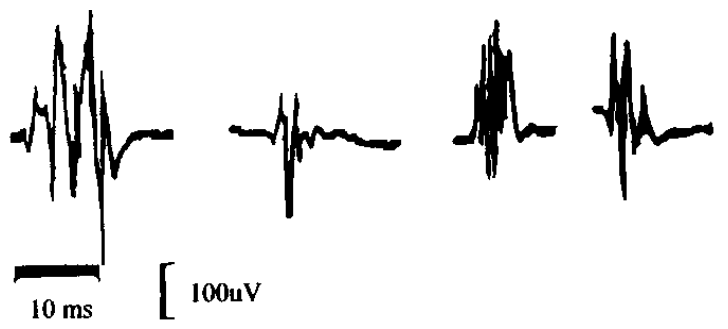

\begin{tabular}{cllll}
\hline AMPLITUDE & DURATION & WAVE FORM & FREQUENCY & SOUND \\
\hline $20-5,000 \mu \mathrm{V}$ & $2-25 \mathrm{~ms}$ & $5-25$ phases & $2-30 \mathrm{~Hz}$ & $\begin{array}{l}\text { Rough/ } \\
\text { Rasping }\end{array}$ \\
\hline
\end{tabular}


Polymyositis: The changes resemble those of myopathy but there are more polyphasic action potentials and sometimes also fibrillation due to involvement of the distal part of the neuron.

Myotonia: Mechanical stimulation of a muscle leads to a repetitive discharge of muscle fibres declining in frequency very rapidly which when conducted to the electromyographic speakers causes the characteristic "diverbomber sound".

Myasthenia gravis: Various abnormalities have been described in EMG in myasthenia gravis, but the chief diagnostic use is to record the improvement in the response produced by an intravenous injection of edrophornium. Before the edrophornium, muscle action potentials, which are initially normal, rapidly progressively decline in amplitude on contraction.

In myasthenic syndrome of Lambert Eaton, though at rates of stimulation of 3 per second the amplitude declines, there is a paradoxical potentiation at higher frequencies though from a lower than normal initial amplitude. Electromyography is thus of value in distinguishing between lesions of the lower motor neurons and the muscle itself. It is a necessary part of the complete examination of a patient with peripheral nerve lesion.

\section{SPECIALISED EMG TECHNIQUES}

Repetetive nerve stimulation: In this test surface electrodes appropriately placed over a particular muscle, records the electrical response of the muscle to supramaximal stimulation of its motor nerve by repetitive, 2-3 Hz shocks delivered before and at selected intervals after maximal voluntary contraction. In normal muscles there is little or no change in size of the compound muscle action potentials (CMAPS) following repetitive stimulation of a motor nerve at 2-3 Hz after voluntary contraction for 20-30 seconds. In myasthenia gravis repetitive stimulation at between 2-5 $\mathrm{Hz}$ may lead to a depression of neuromuscular transmission, with gradual decrement in size of the response recorded from affected muscles. Decrementing responses may also occur in congenital myasthenic syndromes. In Lambert Eaton myasthenic syndrome, the compound muscle action potential elicited by a single stimulus is generally very small. With repetitive stimulation at rates up to $10 \mathrm{~Hz}$ the first few responses may decline in size, but subsequent responses increase. If faster rates of stimulation $20-30 \mathrm{~Hz}$, are used, the CMAPS may increase to several times more than the initial response. In botulism, the response to repetitive stimulation is similar to that in Lambert Eaton syndrome, although with variable findings $(3,4)$.

Single fibre electromyography: In this test a special needle electrode is placed within a muscle and positioned to record action potentials from two muscle fibres belonging to the same motor unit. The time interval between the two potentials is called the neuromuscular jitter. It varies in consecutive discharges. The jitter is normally between 10 and 50 microseconds. This value is increased when neuromuscular transmission is disturbed for any reason. In some cases impulses in individual muscle fibres may fail to occur because of impulse blocking at the neuromuscular junction. Single fibre EMG is more sensitive than repetitive nerve stimulation or determination of acetylcholine antibody levels in diagnosing myasthenia gravis (5-7). Single fibre EMG can also be used to determine the mean number of fibres per motor unit (fibre density) within the recording area and to estimate the number of motor units in a muscle (5).

Active reinnervation is indicated by blocking, increased jitter and increased fibre density. In slow neuropathic processes like juvenile progressive spinomuscular atrophy there is large increase in fibre density, very little blocking and increased jitter. In rapid processes like amyotrophic lateral sclerosis, there is mildly increased fibre density, marked blocking and increased jitter $(6,7)$. In muscular dystrophies there is marked increase in fibre density.

\section{NERVE CONDUCTION STUDIES}

Nerve conduction velocity tests: By recording from the muscle and stimulating its nerve at two different points along its course the velocity of conduction along the intervening segment of the nerve may be calculated. There is a greater slowing of conduction in neuropathies with demyelination than in those with axonal degeneration. Demyelination is present if there is slowing of conduction to more than $30 \%$ of the mean for the population and age group (8-10). The hall mark of axonal loss is reduction in the sensory or motor response amplitudes at all points of stimulation of the nerve. Distal latency and F-response latency are normal. Motor nerve conduction velocity may be reduced or normal. If reduced it is not less than $70-75 \%$ of the normal. The American Association of Electro Diagnostic Medicine has suggested the following criteria for diagnosis:

Suggested nerve conduction studies (NCS) criteria for demyelination:

1. Reduction in conduction velocity to $<70 \%$ of the lower limit of the normal

2. Prolonged distal motor latency to $>130 \%$ of upper limit of the normal

3. Prolonged minimal F- wave latency to $>130 \%$ of the upper limit of the normal

4. Partial conduction block or abnormal temporal dispersion

Features must be present in one or more segments excluding common sites of compression. 
Suggested criteria for conduction block:

Partial conduction block is present if in proximally evoked response:

1. Amplitude is significantly reduced and/or

2. Area of response is significantly reduced

3. There is no substantial increase in negative peak duration

4. There is abnormal temporal dispersion - duration increase by $>30 \%$ of the distal response duration

All these compared to distal evoked response.

For ulnar/median - wrist to elbow segment:

- amplitude drop of $25 \%$ or more

- area decrease of $20 \%$ or more

- duration increase of $25 \%$ or more

For peroneal nerve - knee to ankle segment:

- amplitude drop of $30 \%$ or more

- Area decrease of $25 \%$ or more

- Duration increase of $30 \%$ or more

For tibial nerve changes are the same as for peroneal nerve but amplitude may fall upto 50\%. Age and axonal loss not accounted for.

\begin{tabular}{cclll}
\multicolumn{2}{c}{$\begin{array}{l}\text { Minimal temporal } \\
\text { dispersion }\end{array}$} & $\begin{array}{l}\text { Abnormal temporal } \\
\text { dispersion }\end{array}$ \\
Nerve & $\begin{array}{l}\text { Definite conduction block } \\
\text { Amplitude }\end{array}$ & Area & $\begin{array}{l}\text { Probable conduction block } \\
\text { Amplitude }\end{array}$ & Area \\
Reduction & Reduction & Reduction & Reduction \\
Median $>50 \%$ & $>40 \%$ & $>50 \%$ & $>40 \%$ \\
Ulnar $>50 \%$ & $>40 \%$ & $>50 \%$ & $>40 \%$ \\
Peroneal $>60 \%$ & $>50 \%$ & $>60 \%$ & $>50 \%$ \\
Tibial $>60 \%$ & $>50 \%$ & $>60 \%$ & $>50 \%$
\end{tabular}

Abnormal temporal dispersion is $>30 \%$ increase in duration Distal response amplitude must be $>20 \%$ of lower limit of nolmal (9-13)

In Figure 6, the latency of conduction between stimulus at wrist (distal latency) "a" and the evoked potential in the muscle is 10 msecs. Latency of stimulation at elbow (proximal latency) "b" is 16 msecs. Distance between the stimulating electrodes cathodes is $24 \mathrm{~cm}$. Conduction velocity between elbow and wrist is $24 \mathrm{~cm} / 16-10 \mathrm{~ms}=0.24 \mathrm{~m} / 0.006$ seconds $=$ 40 meters/second.

Conduction velocities can be done for both motor and sensory nerves. In entrapment neuropathies of the peripheral nerves such as carpal tunnel syndrome (median nerve at the wrist) or ulnar nerve compression at the elbow, slowing of conduction velocity occurs across the lesion while it, is normal proximally. The distal latency is significantly delayed and the EMG shows fibrillation potentials and reduced number of action potentials (recruitment) besides the presence of paraesthesia or sensory loss with or without muscle wasting in the nerve distribution areas. The CMAP amplitudes are reduced proximal to the lesion while they are normal distal to the lesion (14-16). Repeated tests may later show improvement. Sensory action potentials are recorded from the median or ulnar nerves at the wrist after stimulating percutaneously the corresponding digital nerves in the fingers with ring electrodes, and from the sural nerve in the lower limb. A loss of potentials or prolonged latency may be found in a sensory neuropathy in the absence of any abnormality of motor fibres.

Quantitative Sensory Testing: This is a new technique in which a stimulator is applied to the nerve distribution area of the skin. Cold or hot temperatures are progressively increased until the patient experiences pain. The temperature sensation and the pain threshold is a measure of the function of small sensory fibres. Abnormalities of this test together with autonomic involvement are usually seen in diabetic neuropathy and other metabolic neuropathies. They can be abnormal while motor conduction velocities are normal.

Segmental electromyography can detect denervation and help the diagnosis of lumbar root lesions caused by the posterior facet joint in which unlike a prolapsed disc, the plain X-ray and myelogram may be entirely normal $(17,18)$.

Figure 6






\section{LATE NERVE RESPONSES}

These are mainly of two types:

(i) The H-reflex is an electrically elicited ankle jerk and can be easily recorded only from resting soleus muscles. It gives information about activity in large afferent (l-alpha) as well as efferent fibres.

(ii) F-response is a late response latency coming after the primary M-response and is evoked every time a motor nerve fibre is stimulated. It is produced by centrifugal discharges from individual motor neurons each of which is initiated by an antidromic volley artificially produced in its axon. It tests conduction in the whole length of an alpha motor axon from the spinal cord, ventral horn to the muscle end plate (2, 19-21). Abnormalities of late responses occur in both primarily axonal and segmental demyelinating neuropathies $(2,22)$ (Figures 7 and 8 ).

Figure 7

H-response

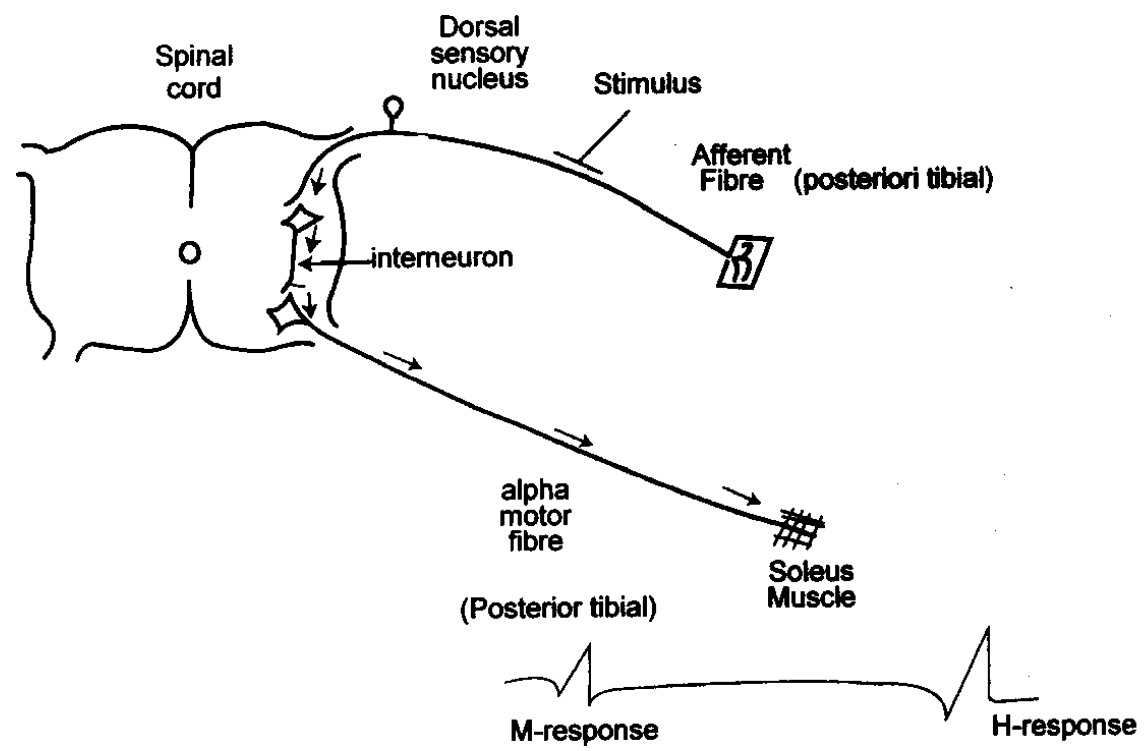

Stimulus sends wave of depolarisation through an afferent fibre of the posterior tibial nerve.

It goes to the alpha motor fibre via the interneuron in the reflex arc to the soleus muscle producing the H-response

Figure 8

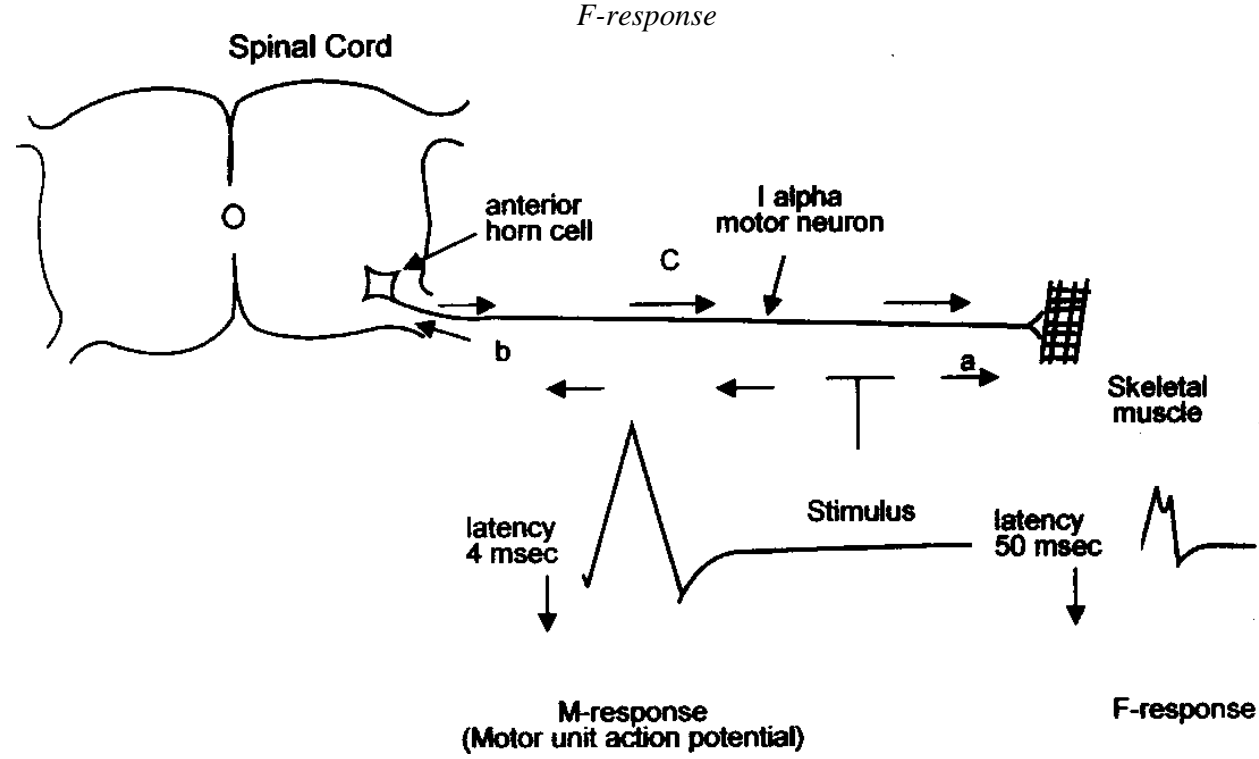

(a) Stimulus sends a wave of depolarisation to the muscle directly producing the M-response

(b) An antidromic volley travels to the cellbody, provoking a wave of depolarisation

(c) Towards the muscle to produce the F-response 
The late responses detect nerve abnormalities at a much earlier stage than the other nerve studies; and are useful not only in evaluation of peripheral neuropathy but also in assessment of entrapment neuropathies and root compression syndromes(23-25). They are either absent or significantly prolonged in entrapment neuropathies and root compression syndrome, and absent in nerve blocks.

\section{NEUROPATHY AND ELECTROPHYSIOLOGICAL TESTS}

Peripheral nerve diseases (neuropathy) are due to:

- complication of a medical disorder

- exposure to toxins and drugs

- genetic abnormalities

- nerve entrapment

- Idiopathic

The causes of neuropathy are many and delineating them can be cumbersome. An undirected search for all possible causes leads to long and expensive investigations which may not give a satisfactory answer.

Frequently no association between cause and effect is found or more than one possible aetiology are identified. The likelihood of accurate diagnosis is high if the patient is approached in an orderly and systematic manner.

The best approach is to first characterise each case into a subtype using the temporal course, the primary pathologic process as revealed by the electrophysiological studies and the pattern of neuropathy. The temporal course may be acute, subacute or chronic, which is obtained from history. The primary pathology may be axonal, demyelinating or compressive. The pattern of neuropathy may be polyneuropathy, mononeuropathy, mononeuritis multiplex, purely sensory or purely motor. This is best determined by electrophysiological studies. In purely sensory neuropathy, electro diagnostic studies help make a distinction between large fibre involvement verses small fibre involvement and autonomic involvement.

With the knowledge of the pattern, temporal course and presence or absence of demyelination, the differential diagnostic possibilities can be considerably narrowed. For example, a chronic symmetric demyelinating sensory motor polyneuropathy that has been present for decades is likely to be a congenital disease. A subacutely evolving multifocal demyelinating neuropathy is usually due to chronic inflammatory demyelinating polyneuropathy. The differentials are few including HIV, paraproteinemia.

If electrophysiologic studies show axonal disorder with mononeuritis multiplex, the list of differentials is shortened to primary or secondary vasculitidis, sarcoidosis and neurofibromatosis. Specific aetiology can then be sought using clues, from history, physical findings and a few selected laboratory tests. If the pathology is nerve compression, the physiologic studies will localise the site and thus guide any necessary further investigations and treatment. Purely sensory neuropathy, is seen in Sjogren's syndrome, sensory variant of CIDP, paraneoplastic neuropathy (anti-Hu antibody), or in congenital lesions. Selective damage to small sensory fibres with decreased pain and temperature sensation with autonomic dysfunction is seen most often in diabetes mellitus. It is also seen in amyloidosis, primary biliary cirrhosis, metronidazole toxicity, leprosy, advanced HIV and hereditary sensory neuropathies.

A purely motor neuropathy may be identified by multifocal conduction block and normal sensory nerves. It has been given the name multifocal motor neuropathy (MMN). It is sometimes associated with high titres of antibodies to GMl ganglioside. Nerve conduction and EMG studies are thus critical in evaluation of peripheral neurologic disease and are an extension of the physical examination. They:

(i) Allow confirmation of the presence of neuropathy helping to differentiate diseases which may present in the same way.

(ii) Allows the physician to asses the pattern of neuropathy.

(iii) Allows determination of axonal disease and dymelination which helps in differential diagnosis.

(iv) By providing information about axonal loss it helps to determine response to therapy and prognosis (26).

\section{EVOKED POTENTIALS}

The most commonly used evoked potentials tests are somatosensory evoked potentials (SSEP), visual evoked potentials (VEP) and auditory evoked potentials (BAEP). Others are cognitive evoked potentials, motor evoked potentials and blink reflexes.

SSEP: Somatosensory evoked potentials tests can be done on either the lower limbs or the upper limbs and either unilaterally or bilaterally. In the lower limbs' SSEP, the peroneal and/or tibial nerves are stimulated at the ankle, or the peroneal nerve may be stimulated at the popliteal fossa.

The responses are collected with electrodes on the spinal cord at the fourth lumbar vertebra (L4) or tenth thoracic vertebra (T10) depending on the level which is being examined or manipulated. The second set of response is obtained with electrodes on the scalp (CZ'Fz) over parietal lobe. The test monitors the integrity of the whole spinocortical pathway from the peripheral nerve, to the spinal cord, to the central pathways (medial lemniscus) to the parietal cortex. It's most common current clinical use is in monitoring the spinal cord state during high risk neuro-surgical procedures $(27,28)$. 
In upper limbs SSEP the median nerve is stimulated at the wrist and response obtained with surface electrodes at Erbs point and scalp (C3' or C4); and at the cervical spine (C7). This test plays a useful role as an adjunct to the EMG examination $(29,30)$.

VEP: In visual evoked potential tests, potentials are elicited by checkered patterns reversal stimuli with the patient seated and concentrating on the stimuli (TV monitor) from a specific distance. A flash of light may also be used for stimulation. Responses are recorded with surface electrodes at the occipital area $(\mathrm{Oz})$ and at the Bregma $(\mathrm{Cz})$. This test is currently used for detection of optic neuritis, multiple sclerosis, retrochiasmal disturbances and for monitoring the visual pathways during surgical procedures in their vicinity (27).

$B A E P$ : In brainstem auditory evoked potential tests each ear is stimulated individually with sound of specific intensity and pitch, while the other ear is blocked. BAEP may be recorded with a single input channel. The responses are picked with ear lobe (Ai,Ao) and scalp surface $(\mathrm{Cz})$ electrodes. Two runs are usually obtained to ensure that the response is replicable and the mean obtained. The test is currently being used in a number of clinical applications. These include detection of brainstem lesions and tumours (multiple sclerosis, acoustic neuromas, cerebellopontine angle tumours and others), brain death and coma, hearing tests and intraoperative monitoring to help ensure that hearing is preserved following certain highrisk neurosurgical procedures (27).

Cognitive Evoked Potential: Some evoked potential components depend upon the mental attention of the subject and the setting in which the stimulus occurs, rather than simply on the physical characteristics of the stimulus. Such event related potentials (ERPs) or endogenous potentials are related in some way to the cognitive aspects of distinguishing an infrequently occurring target stimulus from other events occurring more frequently. The P3 component of the ERP is so far the most important clinically. It is also designated P300 component because of its positive polarity and latency of about 300 to 400 msecs after onset of an auditory stimulus. The P3 component latency is prolonged in many patients with dementia. It is normal in many patients with depression and other psychiatric disorders that might be mistaken for dementia. ERPs are therefore important in diagnosing dementia especially when there is clinical uncertainty. A normal latency response does not exclude dementia $(31,32)$.

Motor Evoked Potentials: In this test, the electrical potentials are recorded from muscle or the spinal cord following stimulation of the motor cortex or central motor pathways. The stimulation is transcutaneous by a magnetic field produced electrically and is painless and safe. Abnormalities in response are produced by several neurologic disorders with clinical or subclinical involvement of central motor pathways, for example, multiple sclerosis, motor neuron disease. The technique provides information important for determining prognosis, and is also useful in monitoring the functional integrity of the central motor tracts intraoperatively (33).

Blink Reflexes: Electrical or mechanical stimulation of the supra orbital nerve on one side leads to two separate reflex responses of orbicularis occuli muscle. An ipsilateral, R1 response having a latency of about 10 msecs and a bilateral R2 response with a latency of about $30 \mathrm{msecs}$. The trigeminal constitute the afferent arm and the facial nerve the efferent arm of the reflex arc. Abnormalities of either nerve or intrinsic lesions of the medulla or pons may lead to unilateral or bilateral loss of response. The findings may therefore be helpful in localising pathology (34).

When nerve conduction studies and EMG are combined with appropriate evoked response tests, the site of lesion can be delineated all the way from the muscle to the spinal cord, brainstem, internal capsule to the cerebral cortex.

The electrophysiological tests are thus of use in electrodiagnostic laboratory as basic clinical screening tools for various diseases of the nervous system, in the operating room as a monitor of CNS integrity during various surgical procedures and in any areas where a quick evaluation of CNS function is needed.

\section{CONCLUSION}

The electrophysiological tests are an extension of a clinical neurological examination as they help to characterise and localise the lesion. They guide in clinching the pathological diagnosis and thus give prognosis. A neurological evaluation would not be complete without them. They should therefore be always done when possible whenever there is a neuromuscular disorder.

\section{REFERENCES}

1. Roger, Bannister. Ancillary investigations, chapter 8, pages 211-234. In: Brain and Bannisters clinical neurology. 7th Edition Oxford University Press, 1992.

2. Amninoff, M. J. Electromyography, Chapter 2, pages 92154. In: Electrodiagnosis in clinical neurology. 2nd Edition Edinburgh, Churchill Livingston, 1987.

3. Dumitru, D. Specialized EMG Techniques, Chapter 4, pages 250-304 Electrodiagnostic Medicine (Eds) Philadelphia, Hanley and Belfus, 1995.

4. Ernest, W. J. Peripheral neuropathies, Chapter 8, pages 246305. Practical EMG, 2nd Edition Baltimore, Williams and Wilkins, 1988.

5. Thick, B. and Stalberg, E. Single fibre EMG findings in polyneuropathy of different etiologies. J. Neurol. Neurosurg. Psychiat. 1974; 37: 403. 
6. Wierchers, D.O. Single fibre EMG with a standard monopolar electrode. Arch. Phys. Med. Rehab. 1985; 66: 47.

7. Stalberg, E. and Thick, B. Motor unit fibre density in extensor digitorum communis muscle. J. Neuro. Neurosurg. Psychiat. 1975; 38: 874-880.

8. Mbuya, S.O. Peripheral neuropathy in a group of AIDS patients at KNH. MMed dissertation, Nairobi University, Department of Internal Medicine, 1994.

9. Oolney, R.K. American Association of Electrodiagnostic Medicine (AAEM) consensus criteria for the diagnosis of partial condition block. Muscle. Nerve. 1999; 22: s225-s229.

10. American Academy of Neurology (AAN) Ad hoc subcommittee, AIDS Task force Criteria for diagnosis of chronic inflammatory demyelinating polyneuropathy. Neurology. 1991; 41: 617- 618.

11. Albers, J. W. and Kelly, J. J. Acquired inflammatory demyelinating polyneuropathies, clinical and electrodiagnostic features. Muscle Nerve 1989;12: 435-451.

12. Barohn, R. J., Kissel, J. T., et al Chronic inflammatory demyelinating polyradiculoneuropathy. Arch. Neurol. 1989; 46: $878-884$.

13. Broomberg, M. B. Comparison of electrodiagnostic criteria for primary demyelination in chronic polyneuropathy. Muscle Nerve 1991; 17: 968-976.

14. Johnson, E.W., Wells R.M. and Duran, R. J. Diagnosis of carpal tunnel syndrome (CPS). Arch. Phys. Med. 1961; 6:137.

15. Thomas, J. E., Lambert, E. H. and Cseur, K. A. Electrodiagnostic aspects of CPS. Arch. Neurol. 1967; 16: 635-641.

16. Wengsam, P. E., John, E. W. and Weirnaman, J. D. CPS; Use of palmer stimulation of sensory fibres. Arch. Phys. Med. Rehab. 1983; 64: 16-19.

17. Swash, M. and Schwartz, M.S. Compression neuropathies, Chapter 3 pages 103-158 in Neuromuscular disease, a practical approach to diagnosis and management 2nd Edition, Springs Berlin 1988.

18. Preston, D. C. and Shapiro B. E. Clinical correlates, Chapter 6 pages 209-227. Electromyography and neuromuscular disorders Published by Butter WorthHeinmann, 1998.

19. Dawson, G. D. and Merton, P. A. Recurrent discharges from motor neurons. Proceedings at the international physiological conference Brussels, 221-222, Brussels St. Catherine Press, 1956.

20. Mayer, R. F. and Fieldman, R. G. Conduction velocity in peripheral nerve during experimental demyelination of the Cat. Neurol. 1964; 14: 714-726.

21. Mcleod, J. G. and Wrays, S. H. An experimental study of the F - wave in the baboon. J. Neurol. Neurosurg. Psychiat. 1966; 29: 196-200.
22. Lachman, T., Shahani, B. T. and Young, R. R. Late responses as aids to diagnosis in peripheral neuropathy. $J$. Neurol. Neurosurg. Psychiat. 1980; 45:156-162.

23. Egloff Baer, S., Shahani B. J. and Young, R. R. Usefulness of the late responses in diagnosis of entrapment neuropathies. Electromyography and Clinical Neurophysiology. 1978; 45:16P.

24. Maccabbee, P. J., Shahani, B. T. and Young, R. R. Usefulness of double simultaneous recording (DSR) and Fresponse studies in diagnosis of carpal tunnel syndrome. Electromyography and Clinical Neurophysiology. 1980; 49:18P.

25. Shahani, B. T., Potts, F., Juguillon, A. and Young, R. R. Maximal-minimal motor nerve conduction and $F$ response studies in normal subjects and patients with ulnar compressive neuropathies. Muscle and Nerve. 1979; 3: 182.

26. Mathew, B. S., Mark, J. B., Steven, L. G. and Arthur, K. A. Neuropathy and the role of electro-diagnostic studies, Chapter 4, pages 65-82. In: Penn Neurology 2000 New York, Alpha Medica Press, 2000.

27. Allen, A., Nuddlean, L. and Starr, A. Assessment of sensory function in the operating room utilizing cerebral evoked potentials: A study of fifty-six surgically anaesthetized patients. Chapter 2, pages 32-61. In: "clinical neurosurgery", N.Y., Waverly press, 1981.

28. Engler, G. L., Spielhols, N. L., Benhard, W. N., Danziger, F., Merking, H., and Wolff, T. Somatosensory evoked potentials during Harrington instrumentation for Scoliosis. J. Bone Joint Surg. 1978; 60-A, 4, 528-532.

29. Grisolia, J. S. and Wiederholt, W. C. Short latency somatosensory evoked potentials from radial, median and ulnar nerve stimulation in man. EEG and Clin. Neurophysiol. 1980; 50: 375-381.

30. Jones, S. J. Investigation of brachial plexus traction lesions by peripheral and spinal somatosensory evoked potentials. J. Neurol. Neurosurg. Psychiat, 1979; 42: 107-116.

31. Baldeweg, T., Gruzelier, J. H., Catlan, J., Pugh, K., et al Auditory visual events related potentials in a controlled investigation of HIV infection. Electroenceph Clin. Neurophysiol. 1993;88: 356-368.

32. Anderer, P., Semlitsch, A. and Saletu, B. "Clinical EEG and ERP studies with nicargoline in degenerative and vascular dementia" pp 697-701. In: Recent advances in event related brain potential research Amsterdam, Elsevier Science. 1996.

33. Aleksander, B. Transcranial electrical and magnetic stimulation, Chapter 48. In: Electromyography, 4th Edition. Philadelphia, Lippincott Williams and Wilkins, 1999.

34. Kimura, J. Evoked potentials, Chapter 5, pages 408-501. In: Electrodiagnosis in disease of nerve and muscle, 2nd Edition Philadelphia, Davis. 1989. 